\title{
Innovation on Classification and Deduction Teaching Methods for a Course
}

\author{
Haozhang Sun \\ School of Art and Design \\ Xi'an University of Technology \\ Xi'an, China
}

\author{
Feng Wang \\ School of Art and Design \\ Xi'an University of Technology \\ Xi'an, China
}

\begin{abstract}
This article, taking folk art curriculum as an example, illuminates the teaching activities should, rooted in the course (folk art) development and existence form, based on the laboring people's living and production, and by digging the main clue of associate attributes of folk art forms, to sort out the teaching perspectives and emphasize the combination of argument and examples; the classification methods applied should focus on respecting and obeying the folk labor life habits, and track the thoughts on the interaction and common characteristics among a variety of folk arts that vary to derivative forms, similar functions and technique connection, etc., with the expectation of exerting positive reference value in the classified recognition during the educational teaching and the improvement of curricular didactics.
\end{abstract}

Keywords-teaching by classification; classification method; deduction

\section{INTRODUCTION}

Folk art comes from laborers, and belongs to laborers. It is a plain and free form of ideographical expression applied and spread among laborers to meet their spiritual needs, rather than the production, labor and social activities. Not classified by the composition of works, techniques or materials, it just shows the pattern of various art forms created by humans in folk. ${ }^{1}$

The existing teaching methods commonly focus on individual introduction to only a few forms and case enumeration, but rarely involve the mutual relations and internal links among lots of folk art forms. For students, folk art seems to only exist in several cases brought to the class, and it is difficult even no way to understand the essence of folk art from people's living and production and widely distributed, so that it can only stay in the simple concept "expression of people's longing for a better life / good luck" and superficial level, or ruleless enumeration of bamboo weaving, straw weaving, batik, clay sculpture, paper-cut, blue calico, folk toys, and so on. ${ }^{2}$ Students' understanding of folk art is an isolated and scattered conceptual term and picture, and even an impression of a few pieces of isolated

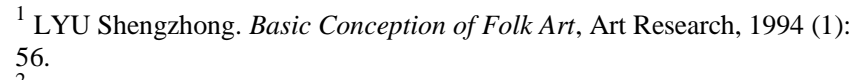

Deng Xingfu. Definition and Classification of Folk Art, Art Observation, 1 997(2): 35 . works of art; therefore, teaching by classification and understanding the nature and origin of folk art are particularly important.

To enable students to thoroughly understand the folk art and become interested and passionate in folk art, the specific teaching practice should track the main clue of relevance among diverse folk art forms and accord with the birth, and development and existence of folk art to achieve the classification teaching of folk art. In the classification approaches, it should be based on the living and production of the working people, respect for compliance with folk labor habits, and strive to achieve objective, plain and realistic, in addition to the use of derivative forms, functional similarity and process connection of folk art and other interrelationships for a more systematic classification of teaching.

The conceptual analysis and explanation of folk art based on relevance and integrality may solve the common problems in folk art courses, such as isolation among various forms and dispersion of knowledge content, and improve the situation that students understanding the categories of folk art only rests on the appearance presented by individual works of art.

\section{FORM DERIVATIVE CLASSIFICATION}

Form derivative classification sets out from the selfcharacteristics of folk art forms to study the relationships among different forms from the functional significances' view before classifying according to its most basic and original form derivative relationship. That is, all folk art forms are strung on the chain of form derivation, allowing establishing the emotional concept of form derivation first, and then fusing into the rational analysis of aesthetic characteristics.

For example, generally in the teaching of folk art will the concrete forms of folk art be listed and introduced separately; and the concrete forms are also based on teachers' materials at hand. The more abundant the data are obtained, the more detailed the corresponding introduction and explanation is; while the explanation and analysis will be relatively rough if lack of information and what students can acquire and understand will become single, one-sided and isolated accordingly, resulting in failure of deep rational 
understanding and the overall perceptual cognizance. In students' impression, the relations among various folk art forms are like a straight aspen forest, independent of and never interfering with one another.

However, in fact, the paper-cut, textile printing, embroidery, fabrics, knitting and other folk art forms are intertwined and interacted. The original form of paper-cut is the draft reference at the beginning of embroidering, known as the paper flower pattern; while textile printing and fabrics are from the coloring \& printing of original fabrics beginning of embroidering, and the shaping of final patterns as living needs and use functions after embroidering, respectively. Technically, it includes a series of processes of spinning, pulp dyeing, sewing, embroidering and knitting, etc. Through the baptism of history, with the improvement of people living conditions, constantly promotion of substance level and spiritual aesthetic needs, numerous links that were in the same chain of production have grown up and, combined with regional cultural differences, eventually formed multiple folk art forms in different styles and features, known as paper-cut, textile printing, embroidery and fabrics. However, the root and opportunity in one another, at the beginning of birth, formed due to the production and labor needs are irreplaceable and inseparable. Given that the above was mostly finished by women at home under the social division of "men for farming and women for weaving" in that time, they were also generally known as "womenwork" or "needlework". The definition of needlework incorporates paper-cut, textile printing, embroidery, fabrics, knitting and other folk art forms seen today, most of which are inherited from mothers to daughters or mother-in-laws to daughter-inlaws, also known as "mother's art."

In the folk art curriculum lecturing and teaching activities should classify according to the folk art derivative forms, and introduce the concept of needlework; besides, explanations on the relevance among a variety of folk art forms are obviously more vivid. Such a meticulous and deep-going way draws the emotional distance between students and folk art closer. Paper-cut, textile printing, embroidery, fabrics, knitting... all these magnificent art forms and works appear no longer far and unreachable, but seemingly from mothers' hands last night. Furthermore, the emotional close inevitably leads to the deepening of understanding and the consolidation of memory. In other words, the strength of perceptual knowledge arouses the profoundness of conceptual understanding, which will greatly improve students' comprehension on folk art and classroom efficiency.

\section{Classification Method Based On Process CONTINUITY}

The classification method based on process continuity refers to: by studying the existing forms of folk art, a shared application medium or a manifestation carrier is expected to be found. In a concrete teaching activity, the establishing procedure of the shared manifestation carrier serves as a

\footnotetext{
${ }^{3}$ Hansheng Editorial Office. Chinese Needlework-
}

Art of Mother, Beijing, Peking University Press, 2006 mainline to link the process sequences together and based on this, characteristics of its form are analyzed in detail. In this way, students can be guided to understand, memorize and deduce relevant folk art forms in virtue of the link-up among process sequences or with the help of the concrete concept of a shared carrier. Therefore, the process sequence of the carrier serves as a reminder of concrete forms and works of different folk art.

For example, among various forms of folk art, stone carving works are mostly attached to building components or annexes such as pillar, lintel, chapiter, plinth, doors and windows, stone tablet, drum-shaped stone blocks, stone lion and stone for tie to a horse. Brick carving works mainly for the decoration of external walls of a building include arch, header cover, lattice window, screen wall, gable, barge course, ridge etc. Wood carving works mainly include doors and windows in residential buildings, door window, tabernacle, internal furniture, etc. The decoration forms of wood carving are mostly on the premise of the realization of its functionality such as openwork carving, relief and full relief. The combination of painting art and wood carving endows the furniture with dual functions of anticorrosion and aesthetics, with the folk features of "carved beams and painted rafters".

People call those working on a field involving a professional skill or those are specialists in a certain field as craftsmen. For instance, those good at stone carving are called as stonemason and those proficient in wood carving as carpenter... In the teaching activity, the word "craftsman" is introduced, and folk art work and its producing process are referred to as "artistic work" or "crafts". By taking construction and decoration of folk architecture as a main line and the labor of the craftsman as threads, folk art processes and forms are expected to be introduced successively in accordance with the procedures of construction and decoration. Based on the connection of "construction" and "craftsman", it is likely to be easier for students to understand and memorize relevant folk art so that they can associate the abstract concept with the concrete construction decoration (decorations and decorating techniques of interior and exterior buildings). Therefore, abstract concepts can be interpreted into concrete pictures in their minds immediately. In this way, all processes and procedures of all kinds of folk art forms such as stone carving, brick carving, wood carving and painting art, are likely to appear successively, vividly and lively, making a strong impression on people, so it will be easier to understand and remember.

\section{Classification Method Based On Similar FUNCTION}

The classification method based on similar function means that by analyzing the functions of folk art forms, to summarize a common similar attribute shared by them. That is, through comparison and induction, a shared or an approximately common functional performance among various folk art forms can be obtained whether in historical function or social function or existing function. Then, based on this, folk art forms with the same or similar functional 
performance can be classified and summarized as one category.

For example, folk art forms including sugar sculpture, clay sculpture, shadow puppet, kite, colored-lantern, etc. appear at different seasons, places and different period of time, that is to say that they are isolated and separated with each other. Thus, it is rather confusing and disturbing just like broken threads if they are mixed and listed with other folk art forms in teaching activity, let alone understanding, memorizing, imitating and taking them as reference.

Through analysis and induction of the characteristics, it can be obtained that: sugar sculpture is a kind of art form existing in streets and lanes. It is made of sugar often through blowing or drawing by a vendor and carried and peddled along streets. Sugar is made into different shapes like flowers, birds, fish and insects to please children. Originated in farm field, clay sculpture is made of clay that is shaped into different forms like Chinese zodiac or other toys which are painted in red and green to imply an auspicious meaning and for children playing. Shadow puppet is an art form performed in front of and behind screen, which is made from skin of pig, cow or donkey which is processed by cutting, carving and drawing to shape historical characters and descript classic stories with the help of lights to create shadows and combining with songs, vocal music and scripts. As one of the important forms of spiritual and culture life of people at old times, it also has an education functions apart from entertaining people. Kite is a kind of folk art form evolved from wood snipe and paper eagle. The first "wood snipe" made by Mo Di then was introduced to people who modified it into modern structure of wood and paper and imitated decorative features of New Year pictures. Kite is a form of entertainment suitable for people of all ages and it is usually played in spring. Colored-lantern is made for celebration often seen in New Year, Festivals or weddings and birthdays. Mostly made of bamboo, paper and silk, it has various types and it is also a culture symbol in addition to its entertainment function.

If folk art forms are classified in accordance with the similar function, it can be found that sugar sculpture, clay sculpture, shadow puppet, kite, colored-lantern etc have a shared function, that is, cultural education and entertainment, so they belong to the domain of culture and entertainment. In this case, the concept of widely used folk words "play goods" can be introduced to summarize the folk art forms with similar function such as sugar sculpture, clay sculpture, shadow puppet, kite and colored-lantern. Under the classification method based on similar function and the concept of "play goods", these scatter folk art forms like sugar sculpture, clay sculpture, shadow puppet, kite, coloredlantern are connected by a shared point and similarity, no longer seemingly disordered, confusing and difficult to memory. As a result, its teaching seems to be more interesting.

In the lecturing and teaching activities of folk art forms, various folk art forms are classified based on their similar function and then integrated with the concept of "play goods" so that they are collected and explained as a category to avoid too scattering. On the basis of the concentration and integration of information, students can find a connecting point of the interrelation among concrete forms of folk art forms, which is much easier to remember. In this way, students are expected to acquire the classification method of information induction and integration when they are trying to understand folk art forms.

\section{CONCLUSION}

Through the reform of classification and deduction method for general folk art courses, students are expected to realize that folk art is originated from and belong to laborers. Folk art is a plain and free expression form created by people themselves in their spare time to satisfy their spiritual life, which is adopted by and popular among people. Folk art is not classified by the constitute form, technique and material but by the characteristics, namely, its functional significance, so the practical function and property shall not be ignored. The characteristics of the aesthetics of folk art shall be made a deep understanding on the basis of a basic acquaintance of its property. Only in this way can people obtain a profound acquaintance and understanding of the achievement of folk art made in aesthetics.

\section{REFERENCES}

[1] LYU Shengzhong. Basic Conception of Folk Art, Art Research, 1994 (1): $56-58$.

[2] Zhang Ningning. Brief Introduction of Folk Art, Art Panorama, 2009 (5): 57

[3] Jin Zhilin. Chinese Folk Art, Art Research, 2003 (3): 63-65.

[4] Yuan Haoxin. Artistic Characteristics of Chinese Folk Art, Decoration, 2005(4): 107.

[5] Wang Lianhai. Glimpse of Folk Art Status Quo, Art Observation, 2007(2): 28. 